\title{
Disruption of coordinated cardiac hypertrophy and angiogenesis contributes to the transition to heart failure
}

\author{
Ichiro Shiojima, ${ }^{1}$ Kaori Sato, ${ }^{1}$ Yasuhiro Izumiya, ${ }^{1}$ Stephan Schiekofer, ${ }^{1}$ Masahiro Ito, ${ }^{2}$ \\ Ronglih Liao, ${ }^{3}$ Wilson S. Colucci, ${ }^{2}$ and Kenneth Walsh' \\ ${ }^{1}$ Molecular Cardiology, ${ }^{2}$ Cardiac Muscle Research Laboratory, and ${ }^{3}$ Myocardial Biology Unit, Whitaker Cardiovascular Institute, \\ Boston University School of Medicine, Boston, Massachusetts, USA.
}

\begin{abstract}
Although increased external load initially induces cardiac hypertrophy with preserved contractility, sustained overload eventually leads to heart failure through poorly understood mechanisms. Here we describe a conditional transgenic system in mice characterized by the sequential development of adaptive cardiac hypertrophy with preserved contractility in the acute phase and dilated cardiomyopathy in the chronic phase following the induction of an activated Akt1 gene in the heart. Coronary angiogenesis was enhanced during the acute phase of adaptive cardiac growth but reduced as hearts underwent pathological remodeling. Enhanced angiogenesis in the acute phase was associated with mammalian target of rapamycin-dependent induction of myocardial VEGF and angiopoietin-2 expression. Inhibition of angiogenesis by a decoy VEGF receptor in the acute phase led to decreased capillary density, contractile dysfunction, and impaired cardiac growth. Thus, both heart size and cardiac function are angiogenesis dependent, and disruption of coordinated tissue growth and angiogenesis in the heart contributes to the progression from adaptive cardiac hypertrophy to heart failure.
\end{abstract}

\section{Introduction}

Heart failure is a final common consequence of various heart diseases and is a leading cause of mortality worldwide $(1,2)$. Hypertrophy of cardiac muscle cells is a common feature of failing myocardium $(3,4)$. Cardiomyocyte hypertrophy in pathological situations such as hypertension, myocardial infarction, or valvular heart disease has been considered to be an adaptive response to increased external load, because hypertrophy can normalize the increase in wall stress induced by mechanical overload. However, increased cardiac mass is clinically associated with increased morbidity and mortality (5), and sustained overload eventually leads to contractile dysfunction and heart failure through poorly understood mechanisms (6-8). Thus, stress-induced or "pathological" cardiac hypertrophy appears to be detrimental for the heart, at least in the chronic phase. On the other hand, normal postnatal growth of the heart or exercise-induced cardiac growth also occur through hypertrophy of individual cardiac muscle cells. These forms of so-called "physiological" cardiac hypertrophy are not associated with contractile dysfunction and are morphologically and molecularly distinct from stress-induced hypertrophy $(9,10)$. These observations raise 2 important questions: (a) what are the mechanisms by which sustained overload results in heart failure? and (b) what determines the difference between physiological and pathological cardiac hypertrophy?

Akt is a serine/threonine protein kinase that mediates cellular growth responses in multiple organisms and cell types (11-13).

Nonstandard abbreviations used: Ang-1, angiopoietin-1; ANP, atrial natriuretic peptide; CsA, cyclosporine A; DOX, doxycycline; DTG, double Tg; HW/BW, heart weight/body weight; $\alpha$-MHC, $\alpha$-myosin heavy chain; $\mathrm{mTOR}$, mammalian target of rapamycin; myrAkt1, myristoylated Akt1; S6K, S6 kinase; tetO, tetracycline operator; tTA, tetracycline transactivator.

Conflict of interest: The authors have declared that no conflict of interest exists.

Citation for this article: J. Clin. Invest. 115:2108-2118 (2005).

doi:10.1172/JCI24682.
There are 3 Akt genes in mammalian genomes (Akt1/PKB $\alpha$, $A k t 2 / P K B \beta$, and $A k t 3 / P K B \gamma$ ), and loss of $A k t 1$ gene in mice leads to general growth retardation and spontaneous apoptosis in restricted cell types $(14,15)$, whereas Akt2 gene disruption results in abnormal glucose metabolism and mild growth deficiency $(16,17)$. However, these genes display a considerable degree of functional overlap, as combined deletion of Akt 1 and Akt2 genes results in perinatal lethality with severe growth retardation and multiple developmental defects (18). In the heart, Akt is an important positive regulator of normal postnatal cardiac growth (19) and is also activated by exercise training (20-22), by pressure overload (23), and in diseased human hearts (24). In addition, overexpression of activated Akt1 or Akt3 in the heart under the control of $\alpha$-myosin heavy chain $(\alpha-\mathrm{MHC})$ promoter is sufficient to induce cardiac hypertrophy and, in some cases, contractile dysfunction in Tg mice (25-28). These data suggest that Aktdependent signaling pathways might be involved both in normal (or physiological) and in abnormal (or pathological) cardiac growth. However, how a single signaling molecule differentially mediates both normal and abnormal cardiac growth is unclear.

To examine the mechanisms controlling the progression from adaptive hypertrophy to failure, we have developed a Tg system to switch Akt signaling on or off in the heart. Here, we show that cardiac-specific inducible Akt $1-\mathrm{Tg}$ mice develop adaptive or physiological hypertrophy following short-term induction but exhibit pathological hypertrophy and cardiomyopathy with longer periods of transgene induction. Coronary angiogenesis is enhanced in the acute phase of heart growth but impaired in the chronic phase, and the inhibition of angiogenesis during the initial phase of heart growth results in accelerated contractile dysfunction and impaired cardiac growth. These data suggest that disruption of coordinated tissue growth and angiogenesis in the heart contributes to the progression from adaptive cardiac hypertrophy to heart failure. 


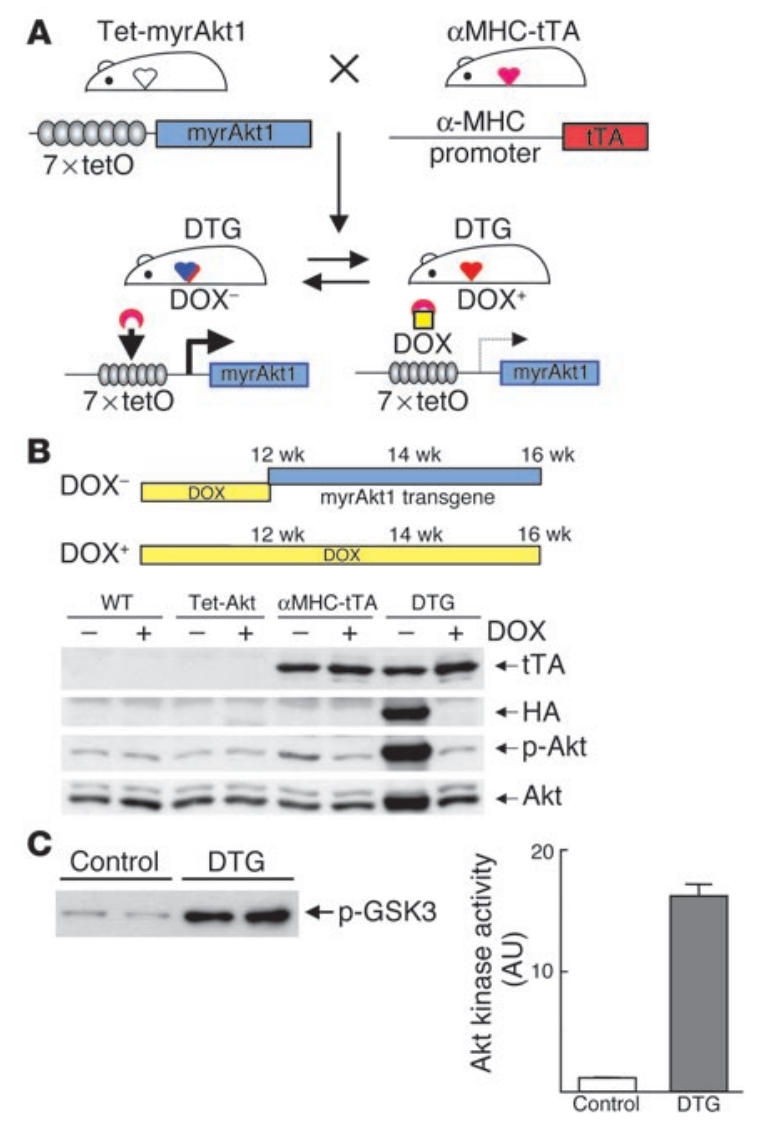

\section{Results}

Generation of cardiac-specific inducible Akt1-Tg mice. Two lines of $\mathrm{Tg}$ mice (Tet-myrAkt 1 and $\alpha \mathrm{MHC}$-tTA) were used to generate cardiacspecific inducible Akt1-Tg mice (Figure 1A). The Tet-myrAkt1 line harbors an active myristoylated form of Akt 1 (myrAkt1) transgene under the control of multimerized tetracycline operator (tetO) sequences, and the $\alpha \mathrm{MHC}$-tTA line expresses tetracycline transactivator (tTA, a fusion protein of tetO-binding domain and VP16 transactivation domain) in the heart driven by $\alpha$-MHC promoter (29). Treatment of double Tg (DTG) mice harboring both transgenes with doxycycline (DOX) results in repression of myrAkt1 expression because DOX associates with tTA and inhibits its binding to tetO sequences. On the other hand, withdrawal of DOX results in tTA binding to tetO elements and induction of myrAkt1 expression in the heart.

Mating of Tet-myrAkt 1 mice and $\alpha M H C$-tTA mice resulted in the generation of mice with 4 different genotypes (WT, Tet-myrAkt1 $\mathrm{Tg}, \alpha \mathrm{MHC}$-t TA Tg, and DTG) at the expected frequencies. To examine the regulated expression of Akt 1 transgene, we divided these mice into $\mathrm{DOX}^{-}$and $\mathrm{DOX}^{+}$groups. The $\mathrm{DOX}^{-}$group was treated with DOX in drinking water until the age of 12 weeks, which was followed by DOX withdrawal for 4 weeks; and the DOX ${ }^{+}$ group was treated with DOX until the age of 16 weeks (Figure 1B). Western blot analysis of heart lysates harvested at 16 weeks of age revealed that transgene expression detected by anti-HA blot was observed only in the DOX- DTG mice, which indicated that the expression of Akt 1 transgene in the heart is tightly regulated in a DOX-dependent manner. The induced expression of Akt 1 transgene was associated with marked increase in phosphorylation

\section{Figure 1}

Generation of cardiac-specific inducible Akt1-Tg mice. (A) Schematic illustration of binary Tg system. (B) DOX-dependent expression of Akt1 transgene. Top: Temporal profile of DOX treatment. Bottom: Western blot analysis of transgene expression. (C) Kinase assay in vitro. Left: Western blot analysis of Akt substrate. GSK3, glycogen synthase kinase 3. Right: Densitometric analysis of Akt activity.

levels of Akt at Ser473, moderate increase in total Akt protein levels, and increase in Akt kinase activity in the heart (Figure 1, B and C). We also established a second Tet-myrAkt1 Tg mouse line that exhibits relatively lower levels of Akt 1 transgene expression (data not shown). Because the first Tet-myrAkt1 line exhibited higher levels of transgene expression and permitted a more rapid assessment of cardiac growth and function, further experiments were performed using this line of Tet-myrAkt 1 mice.

Short-term Akt activation induces reversible bypertrophy. To investigate the consequences of transient Akt activation in the adult heart, we induced transgene expression at 12 weeks of age, maintained it for 2 weeks, and repressed it by restarting DOX treatment (Figure 2A). Activation of Akt signaling for 2 weeks induced an approximately $80 \%$ increase in heart weight/body weight (HW/BW) ratio. This increase was completely reversible, and heart size returned to the basal level 2 weeks after DOX treatment was restarted (Figure 2, $\mathrm{A}$ and $\mathrm{B}$ ). The time course of transgene expression and $\mathrm{HW} / \mathrm{BW}$ ratio was also examined (Figure $2 \mathrm{C}$ ). Transgene expression was first detected at day 7 , which indicated that the initial transgene expression occurred sometime between day 3 and day 7 . Transgene expression reached its maximal level on day 12 and was maintained at a high level at day 14, when DOX was readministered. Marked repression of transgene expression was observed as early as 1 day after DOX treatment was restarted, and transgene expression was completely suppressed by day 3 after DOX treatment was restarted. Changes in HW/BW paralleled the changes in transgene induction (Figure 2C). Although the HW/BW ratio was slightly increased at day 10 , this increase was not statistically significant. However, there was a robust increase in HW/BW ratio at day 12, which was maintained at day 14 . When DOX was readministered, significant repression of hypertrophy was observed as early as 1 day after DOX treatment was restarted, and a nearly complete reversal of HW/BW ratio was observed by day 3 after DOX treatment was restarted. As revealed by echocardiography, induction of Akt 1 transgene resulted in a significant increase in posterior wall thickness without changing LV end-diastolic dimension or contractile function as shown by percent fractional shortening (Figure 2D). As revealed by histology, there was an increase in individual myofiber size, which was also reversed after transgene repression (Figure 2E). There was no interstitial fibrosis and no induction of fetal-type cardiac genes such as atrial natriuretic peptide (ANP) or $\beta$-MHC (Figure 2, E and F). Protein levels of SERCA2, as determined by Western blot analysis, were not altered (data not shown). These data suggest that cardiac hypertrophy produced by short-term Akt transgene activation is similar to a physiological form of cardiac growth.

Sustained Akt activation induces extensive bypertrophy and cardiac dysfunction. The consequences of prolonged Akt activation in the heart were also examined. Maintaining the transgene expression for 6 weeks induced markedly greater cardiac hypertrophy $(\sim 170 \%$ increase in HW/BW ratio) compared with short-term transgene induction (Figure 3, A and B). When transgene expression was maintained for 6 weeks and then repressed for 2 weeks, the increase 


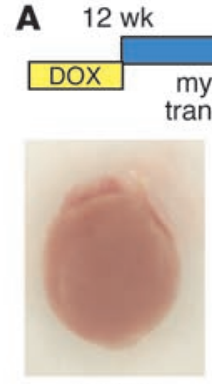

12 wk
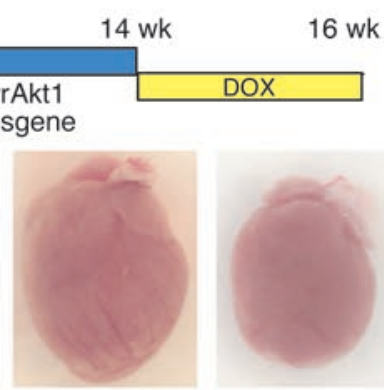

14 wk

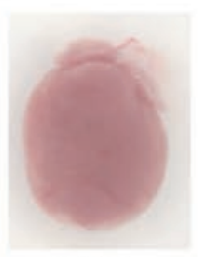

16 wk
B

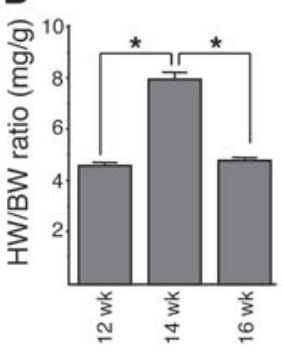

C
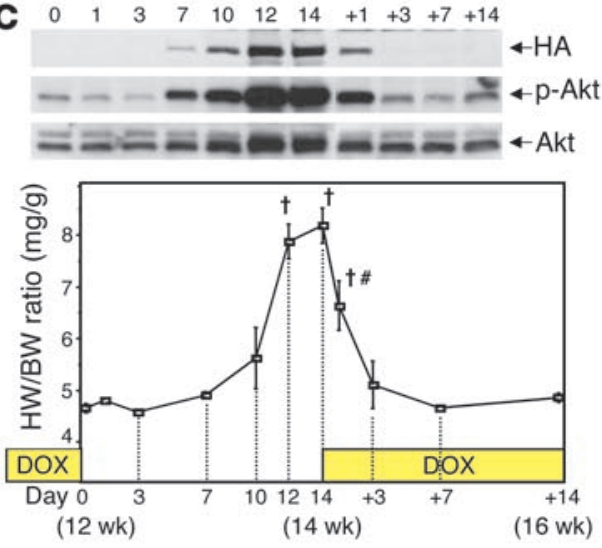

E
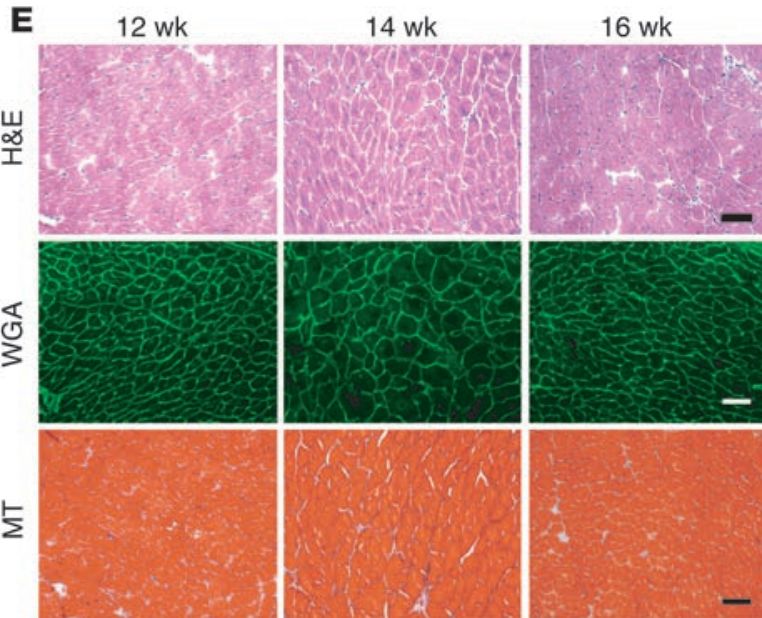

Figure 2

Cardiac hypertrophy is induced by short-term Akt activation. (A) Top: Temporal profile of DOX treatment. Bottom: Representative gross morphology of the DTG hearts. (B) HW/BW ratio. ${ }^{*} P<0.01$. (C) Time course of cardiac hypertrophy. Top: Time course of transgene expression. Bottom: Time course of HW/BW ratio. ${ }^{\dagger} P<0.01$ versus day 0 ; $\# P<0.05$ versus day 14. (D) Echocardiography. Top: Representative M-mode recordings. Bottom: Posterior wall thickness (PWT), LV end-diastolic dimension (LVDd), and percent fractional shortening (\%FS). ${ }^{* *} P<0.05$. (E) Histological analysis. H\&E, wheat germ agglutinin (WGA), and Masson's trichrome (MT) staining of heart sections. Scale bars: $50 \mu \mathrm{m}$. (F) Fold induction of ANP and $\beta$-MHC expression after short-term Akt activation.

in $\mathrm{HW} / \mathrm{BW}$ ratio was only partly reversed (the $\sim 170 \%$ increase in $\mathrm{HW} / \mathrm{BW}$ ratio decreased to a $\sim 70 \%$ increase relative to the level in mice maintained on DOX). According to echocardiography, there was evidence of LV dilatation and contractile dysfunction after 6 weeks of transgene induction (Figure 3C). Intriguingly, repression of Akt transgene for 2 weeks after prolonged induction resulted in a further increase in LV diameter and further impairment of contractility (Figure 3C). As revealed by histology, Akt activation for 6 weeks induced an increase in myofiber size and interstitial fibrosis. After transgene expression was repressed, the increase in myofiber size was partly reversed, while interstitial fibrosis was not (Figure 3D). Finally, there was a significant increase in the expression levels of ANP and $\beta$-MHC genes after chronic Akt activation (Figure 3E). Transgene induction for 4 weeks also induced mild LV dilatation and contractile dysfunction with interstitial fibrosis, and maintaining transgene expression for more than 6 weeks resulted in lethality of the animals, with mortality rate more than $50 \%$ at 8 weeks (summarized in Table 1). These findings indicate that, although short-term Akt activation leads to the physiological form of cardiac hypertrophy, prolonged Akt activation results in pathological hypertrophy that is detrimental for heart function.

Rapamycin prevents Akt-mediated contractile dysfunction. If extensive cardiac growth is responsible for the detrimental effect of the Akt1 transgene on the heart, we reasoned that selective inhibition of hypertrophy in the setting of prolonged Akt activation should prevent the development of contractile dysfunction. Because the mammalian target of rapamycin (mTOR) branch of Akt signaling has been implicated in Akt-mediated enhanced protein synthesis and cell growth $(25,30)$, Akt 1 transgene expression was induced for 6 weeks while animals were treated with the mTOR inhibitor rapamycin. Rapamycin treatment almost completely inhibited Akt-mediated cardiac growth both in the acute and chronic phases (Figure 4A), which suggests that the growth-promoting effect of Akt is largely mTOR dependent. Western blot analysis indicated that increased p70 S6 kinase (S6K) phosphorylation was completely inhibited by rapamycin after short-term Akt activation (Figure 4B, left). However, phosphorylation levels of S6K in DTG hearts were not altered in the chronic phase of Akt induction, which indicates that Akt to S6K signaling is impaired following prolonged Akt 


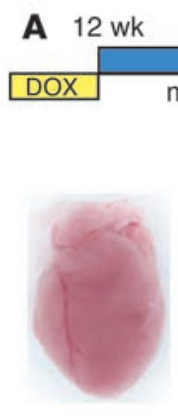

12 wk

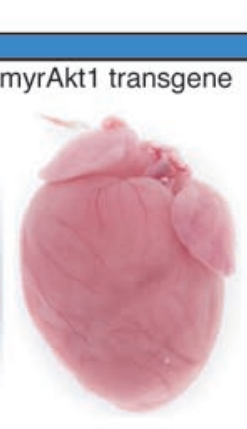

$18 w k$
$18 w k \quad 20 w k \quad \mathbf{B}$

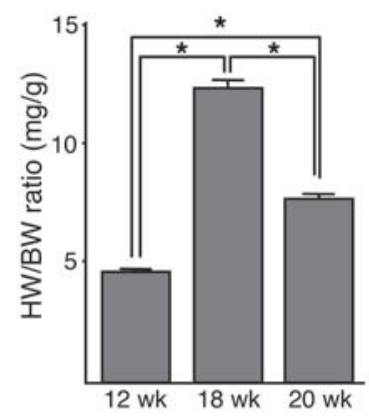

C 12 wk

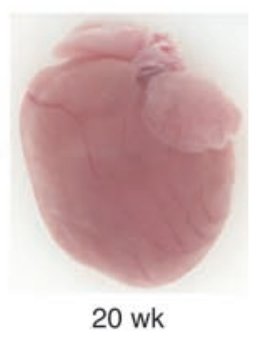

$18 \mathrm{wk}$
20 wk

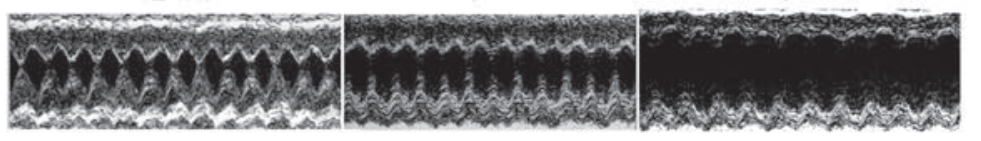

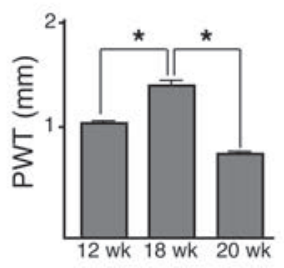
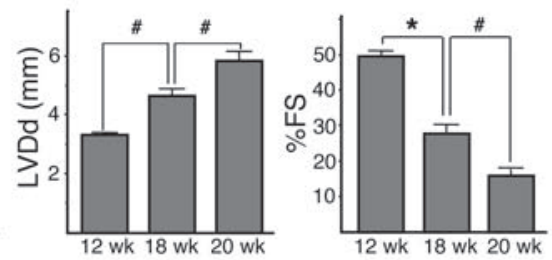

D
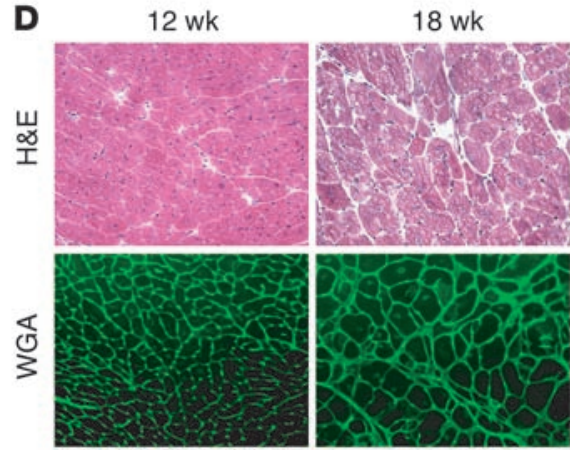

$18 w k$

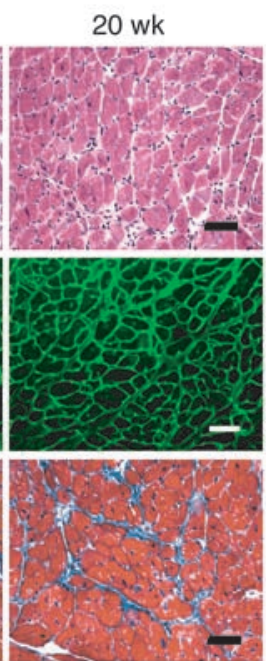

E

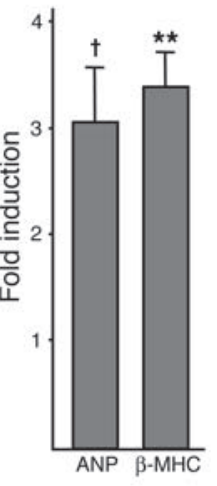

Figure 3

Extensive hypertrophy and contractile dysfunction induced by prolonged Akt activation. (A) Top: Temporal profile of DOX treatment. Bottom: Representative gross morphology. (B) HW/BW ratio. ${ }^{\star} P<0.01$. (C) Echocardiography. Top: Representative M-mode recordings. Bottom: posterior wall thickness, LV end-diastolic dimension, and percent fractional shortening. ${ }^{*} P<0.01$; ${ }^{*} P<0.05$. (D) Histology: H\&E, wheat germ agglutinin, and Masson's trichrome staining of heart sections. Scale bar: 50 $\mu \mathrm{m}$. (E) Fold induction of ANP and $\beta$-MHC expression after prolonged Akt activation. ${ }^{* *} P<0.01$ versus control; $\uparrow P<0.05$ versus control.

altered following short-term Akt activation ( 2 weeks) but was significantly reduced after long-term Akt activation (6 weeks) in cardiac muscle cells (Figure 5A). Because there was an approximately $80 \%$ and approximately $170 \%$ increase in HW/BW ratio after short-term and long-term Akt activation, respectively, these data indicate that coronary angiogenesis initially keeps pace with cardiac growth but is impaired during the extensive growth that results from prolonged Akt activation. Next, we examined the expression of a panel of angiogenic growth factors in these hearts. Although there was no change in the protein levels of fibroblast growth factor 2, angiopoietin-1 (Ang-1), and sonic hedgehog between control and DTG hearts (data not shown), we found that protein levels of VEGF-A and Ang-2 were increased in DTG hearts after transient but not prolonged myocardial Akt activation (Figure 5B). These data suggest that the coordinated induction of VEGF-A and Ang- 2 by Akt activation in the myocardium promotes coronary angiogenesis in the early phase of cardiac growth, and failure to maintain the expression of these growth factors contributes to impaired coronary angiogenesis and contractile dysfunction in the chronic phase.

Because treatment with rapamycin prevented contractile dysfunction after prolonged Akt activation in the heart (Figure 4B, right). Echocardiography measurements revealed that rapamycin completely inhibited the increase in wall thickness and LV dimension and decrease in contractility that resulted from prolonged Akt activation (Figure 4C). The increases in myofiber size, interstitial fibrosis, and fetal gene expression induced by prolonged Akt activation were also inhibited by rapamycin treatment (Figure 4, D and E). Thus, both contractile dysfunction and pathological remodeling resulting from prolonged Akt activation were inhibited by rapamycin treatment.

Coronary angiogenesis during Akt-mediated cardiac growth. To investigate the potential mechanisms by which extensive hypertrophy detrimentally affects the myocardium, we examined the extent of coronary angiogenesis during Akt-mediated cardiac growth. Examination of capillary density by CD31 staining in control and DTG hearts revealed that capillary density was not activation (Figure 4), we examined capillary density and the expression of VEGF-A and Ang-2 in rapamycin-treated animals. After short-term Akt activation, there was no significant change in capillary density between rapamycin-treated and vehicle-treated DTG hearts (Figure 5C, left). However, rapamycin treatment inhibited the decrease in capillary density that was observed after long-term Akt activation in vehicle-treated DTG animals (Figure $5 \mathrm{D}$, left). Rapamycin treatment also completely suppressed the induction of VEGF-A and Ang-2 after short-term myocardial Akt activation (Figure 5C, right), consistent with the notion that induction of these growth factors by Akt is mTOR dependent (31). Rapamycin had little effect on the expression of VEGF-A and Ang-2 at the chronic stage of cardiac growth, when these growth factors are not upregulated in DTG relative to control hearts (Figure 5D, right). Taken together, these data suggest that myocardial Akt 

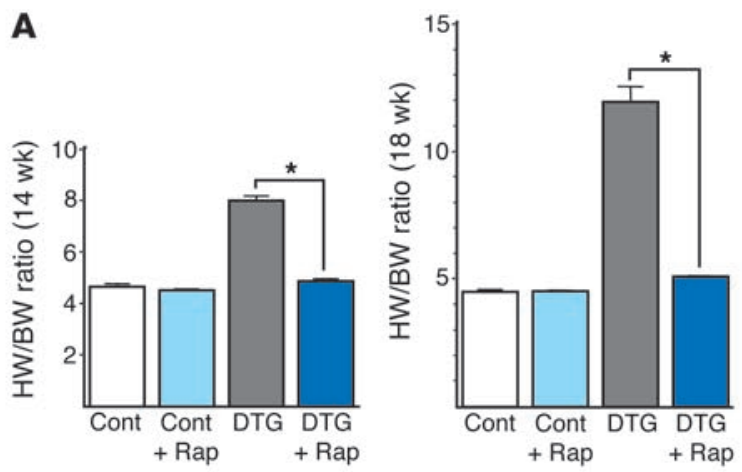

B

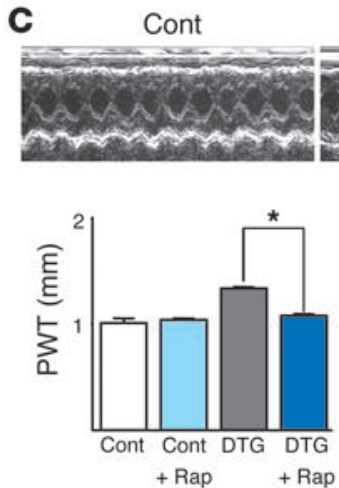

Cont + Rap
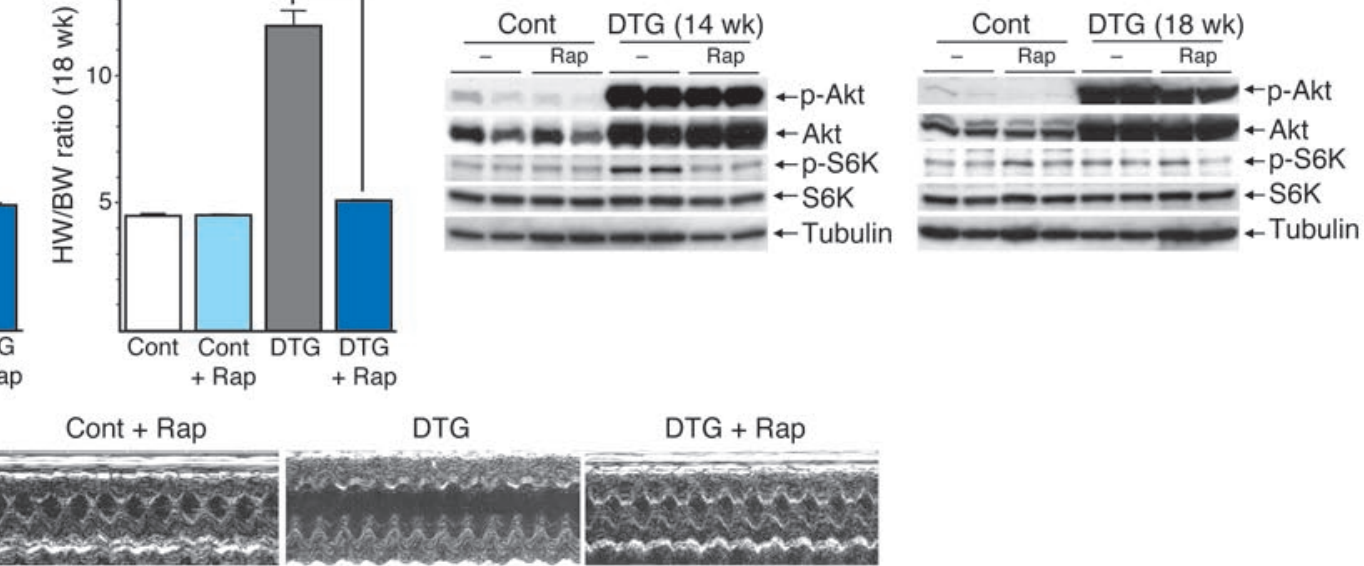

DTG

$\mathrm{DTG}+\mathrm{Rap}$
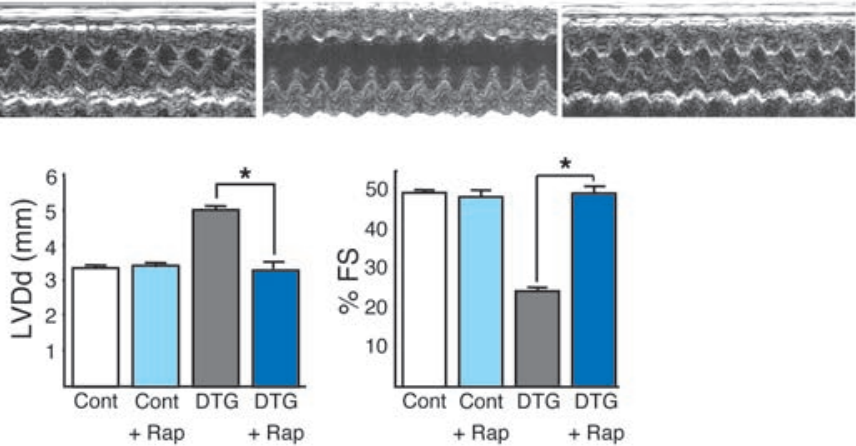

D

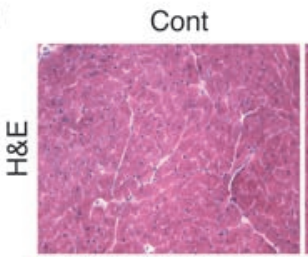

Cont + Rap
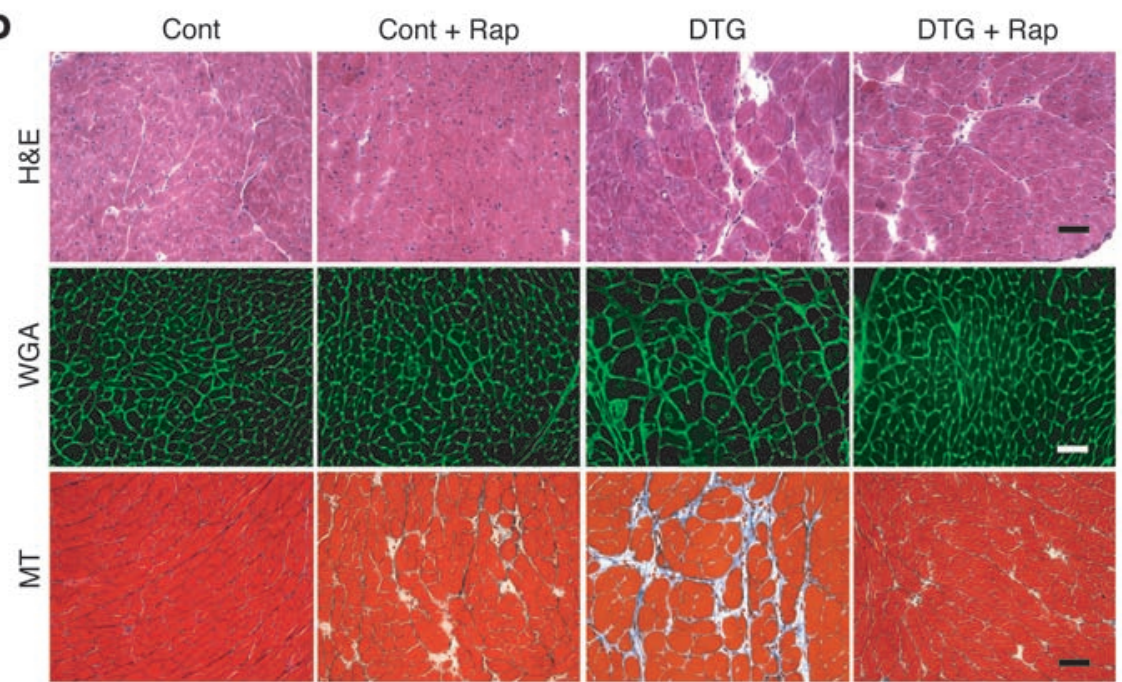

E
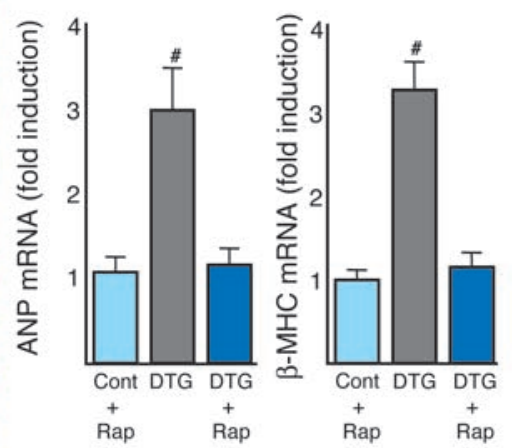

\section{Figure 4}

Prevention of cardiac growth and heart failure progression by rapamycin. (A) HW/BW ratio of mice in the acute (left) and chronic (right) phase after Akt transgene induction. ${ }^{*} P<0.01$. (B) Western blot analysis of Akt transgene and S6K phosphorylation in the acute (left) and chronic (right) phase after Akt transgene induction. (C) Echocardiography. Top: Representative M-mode recordings. Bottom: posterior wall thickness, LV end-diastolic dimension, and percent fractional shortening 6 weeks after transgene induction. ${ }^{*} P<0.01$. (D) Histological analysis. H\&E, wheat germ agglutinin, and Masson's trichrome staining of heart sections 6 weeks after transgene induction. Scale bar: $50 \mu \mathrm{m}$. (E) Fold induction of ANP and $\beta-\mathrm{MHC}$ expression. ${ }^{\#} P<0.05$ versus control. Cont, control; Rap, rapamycin treatment.

activation initially enhances coronary angiogenesis via mTORdependent induction of VEGF-A and Ang-2 and that failure to maintain the expression of these growth factors due to defective Akt/mTOR signaling contributes to the impaired coronary angiogenesis and contractile dysfunction that are observed following extensive growth of the heart.

Akt/mTOR signaling mediates angiogenic growth factor induction in vitro. Since rapamycin completely inhibits Akt-mediated cardiac growth, the inhibitory effects of rapamycin on VEGF-A and Ang-2 induction might be secondary to the inhibition of cardiac growth rather than due to the inhibition of mTOR-dependent signaling. To further assess whether the induction of VEGF-A and Ang-2 by Akt is mTOR dependent, we infected cultured adult rat cardiac myocytes with an adenoviral vector encoding constitutively active Akt1 and examined expression levels of VEGF-A and Ang-2. Overexpression of Akt 1 was found to enhance the expression 
A
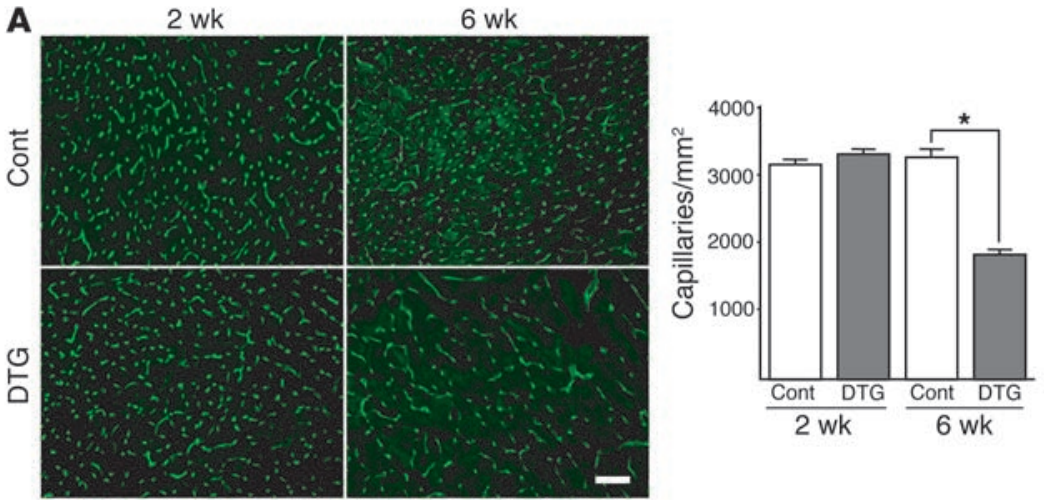

B

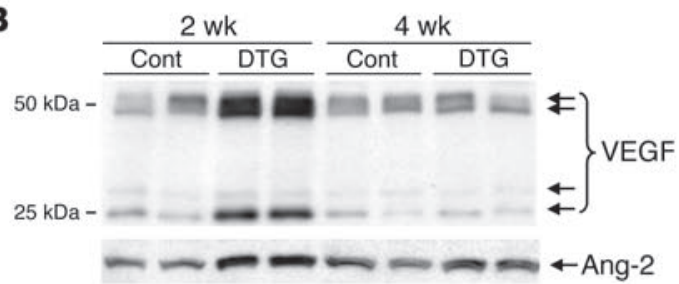

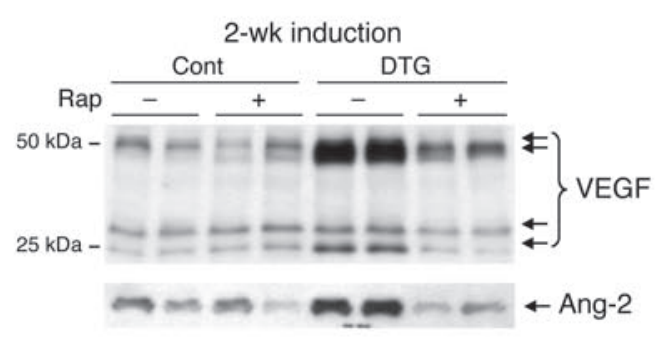

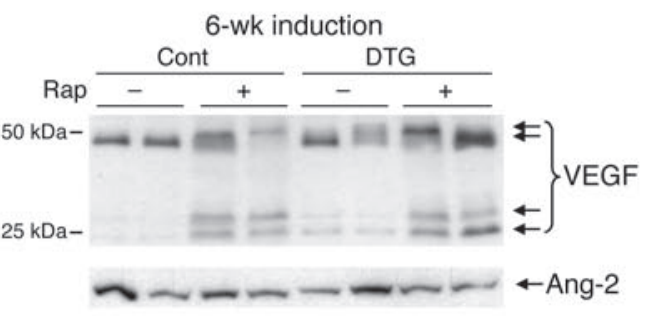

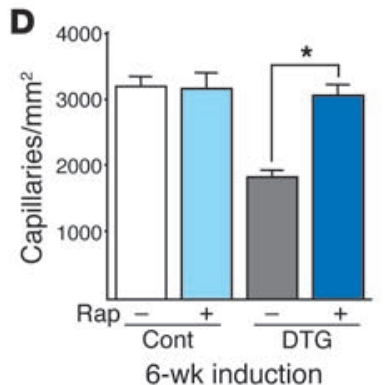

6-wk induction

\section{Figure 5}

Coronary angiogenesis and angiogenic growth factor expression. (A) Left: Representative CD31 staining. Scale bar: $50 \mu \mathrm{m}$. Right: Capillary density. ${ }^{*} P<0.01$. (B) Expressions of VEGF-A and Ang-2 in the heart. (C) Effects of rapamycin on capillary density and angiogenic growth factor expression in the acute phase. Left: Capillary density after 2 weeks of transgene induction. Right: Expression of VEGF-A and Ang-2 after 2 weeks of transgene induction. (D) Effects of rapamycin on capillary density and angiogenic growth factor expressions in the chronic phase. Left: Capillary density after 6 weeks of transgene induction. ${ }^{*} P<0.01$. Right: Expression of VEGF-A and Ang-2 after 6 weeks of transgene induction.

Fc fragment (Figure 7A, top) and is a potent angiogenesis inhibitor that acts as a decoy VEGF receptor (32). While Akt activation for 2 weeks resulted in an increase in wall thickness without changing LV dimension or contractile function, the simultaneous overexpression of Flk1-Fc and the Akt1 transgene resulted in contractile dysfunction associated with $\mathrm{LV}$ dilatation (Figure 7A). Flk1-Fc also suppressed the Akt-induced increase in wall thickness and resulted in partial inhibition of cardiac growth (Figure 7, A and B). These data indicate that inhibition of coronary angiogenesis during Akt-mediated cardiac hypertrophy is sufficient to induce contractile dysfunction. They also indicate that Akt-mediated cardiac growth is angiogenesis dependent. Western blot analysis showed that overexpression of Flk1-Fc had no effect on expression levels of the Akt1 transgene, S6K phosphorylation, or Akt-mediated Ang-2 induction (Figure 7C). Interestingly, however, Flk1-Fc overexpression resulted in massive induction of VEGF-A in the myocardium both in control and DTG hearts (Figure 7C), which suggests the existence of a negative feedback mechanism whereby VEGF signaling inhibits its own expression in the myocardium. Histological analysis revealed interstitial fibrosis and reduced

of Ang-2 in cultured myocytes (Figure 6A) and increased the release of VEGF-A in the culture media (Figure 6B). Treatment of Akt1-infected myocytes with rapamycin partially inhibited the induction of VEGF-A and Ang-2, although Akt-induced S6K phosphorylation was completely suppressed by rapamycin (Figure $6 \mathrm{~A})$. These data suggest that Akt-mediated induction of VEGF-A and Ang-2 in the heart is at least in part mTOR dependent.

Inbibition of angiogenesis results in contractile dysfunction and impaired cardiac growth. Because contractile dysfunction after prolonged Akt activation was associated with impaired coronary angiogenesis and reduced capillary density, we examined whether impaired angiogenesis is sufficient to induce contractile dysfunction. For this purpose, an adenoviral vector encoding Flk1-Fc or a control vector was injected intravenously, and the Akt1 transgene was induced for 2 weeks (Figure 7A, top). Flk1-Fc is a soluble fusion protein of a ligand-binding domain of Flk1 and a murine IgG2 $\alpha$ capillary density in Flk1-Fc-treated DTG mice (Figure 7, D and E). These observations indicate that the inhibition of physiological growth-associated coronary angiogenesis results in conversion from physiological to pathological hypertrophy.

\section{Discussion}

In the present study, we established a conditional $\mathrm{Tg}$ system in which physiological and pathological cardiac hypertrophy can be induced in a sequential manner in mice. Using this model system, we have shown that physiological cardiac growth with preserved contractile function is associated with enhanced coronary angiogenesis, whereas pathological cardiac hypertrophy with reduced contractility is accompanied by impaired coronary angiogenesis. We have also shown that mTOR-dependent expression of VEGF-A and Ang-2 is upregulated during the physiological phase of cardiac growth but downregulated during the pathological phase of 


\section{Table 1}

Summary of phenotypes of conditional Akt1 TG mice

\begin{tabular}{|c|c|c|c|c|c|c|c|}
\hline \multirow{2}{*}{\multicolumn{2}{|c|}{ HW/BW ratio }} & 2 wk on & 2 wk on, 2 wk off & 4 wk on & 4 wk on, 4 wk off & 6 wk on & \multirow{2}{*}{$\begin{array}{c}\mathbf{6} \text { wk on, } 2 \text { wk of } \\
\sim 170 \%\end{array}$} \\
\hline & & $\sim 180 \%$ & $\sim 100 \%$ & $\sim 200 \%$ & $\sim 120 \%$ & $\sim 270 \%$ & \\
\hline UCG & $\begin{array}{l}\text { LVDd } \\
\text { PWT } \\
\% \text { FS }\end{array}$ & $\underset{\uparrow}{\rightarrow}$ & $\begin{array}{l}\rightarrow \\
\rightarrow \\
\rightarrow\end{array}$ & $\begin{array}{l}\uparrow \\
\uparrow \\
\downarrow\end{array}$ & $\begin{array}{c}\rightarrow \sim \uparrow \\
\rightarrow \\
\rightarrow \sim \downarrow\end{array}$ & $\begin{array}{c}\uparrow \uparrow \\
\uparrow \\
\downarrow \downarrow\end{array}$ & $\begin{array}{c}\uparrow \uparrow \uparrow \\
\downarrow \\
\downarrow \downarrow \downarrow\end{array}$ \\
\hline $\begin{array}{l}\text { Fibro } \\
\text { Survi }\end{array}$ & $\stackrel{-}{\text { rate100\% }}$ & - & $\stackrel{+}{+}$ & $\begin{array}{c}+ \\
100 \%\end{array}$ & $\begin{array}{c}++ \\
90 \%\end{array}$ & $\begin{array}{c}++ \\
55 \%\end{array}$ & \\
\hline
\end{tabular}

The number of weeks that the transgene was on and/or off is indicated. AContractility is improved when transgene is expressed in failing hearts. \%FS, percent fractional shortening; LVDd, LV end-diastolic dimension; PWT, posterior wall thickness; UCG, ultrasound cardiography; $\rightarrow$, no change; $\uparrow$, increase; $\uparrow \uparrow$, strong increase; $\uparrow \uparrow \uparrow$, very strong increase; $\sim \uparrow$, slight increase; $\rightarrow \sim \uparrow$, no change followed by slight increase; $\downarrow$, decrease; $\downarrow \downarrow$, strong decrease; $\downarrow \downarrow \downarrow$, very strong decrease; $\sim$, slight decrease; $\rightarrow \sim \downarrow$, no change followed by slight decrease; - , negative; +, positive; ++, strongly positive.

cardiac hypertrophy. Furthermore, inhibition of coronary angiogenesis during the physiological growth phase results in impaired cardiac growth, contractile dysfunction, and an accelerated conversion from physiological to pathological hypertrophy. Collectively, these data suggest that both heart size and cardiac function are angiogenesis dependent and that disruption of coordinated tissue growth and angiogenesis in the heart contributes to the progression from adaptive cardiac hypertrophy to heart failure.

The physiological nature of cardiac hypertrophy induced by shortterm Akt activation is demonstrated by the following observations: (a) preserved contractile function, (b) lack of interstitial fibrosis, (c) lack of fetal cardiac gene induction, and (d) completely reversible hypertrophy. However, pathological cardiac hypertrophy associated with interstitial fibrosis, induction of fetal genes, LV dilatation and contractile dysfunction occurred upon long-term Akt induction that led to extensive cardiac growth. Thus, both physiological and pathological cardiac hypertrophy, although morphologically and molecularly distinct from each other, can be induced in a sequential manner by the activation of a single signaling pathway as heart weight increases from $180 \%$ to $270 \%$ in DTG relative to control mice. Intriguingly, contractile dysfunction was further impaired in the failing hearts when Akt was switched off after prolonged activation (Figure 3), which indicates that Akt signaling contributes to maintaining contractile function even at this late stage. Thus, long-term Akt activation induces contractile dysfunction in spite of its beneficial effects on contractility, which suggests that excessive cardiac growth, rather than prolonged Akt activation itself, is detrimental for the heart. This notion is further supported by the following observations: (a) suppression of cardiac growth by rapamycin prevented heart failure progression induced by longterm Akt activation (Figure 4); (b) prolonged but lower levels of Akt expression for 6 weeks using a different line of Tet-myrAkt 1 mice resulted in moderate cardiac hypertrophy (an approximately 35\% increase in $\mathrm{HW} / \mathrm{BW}$ ratio) with preserved contractile function (data not shown); and (c) conventional cardiac-specific Akt1 TG mice that show approximately $40 \%$ increase in heart size do not exhibit any defect in contractility (27), whereas those that show approximately

\section{Figure 6}

Induction of VEGF-A and Ang-2 by Akt in cultured adult cardiac myocytes. (A) Top: Representative Western blot analysis of Ang-2, Akt, and S6K. Bottom: Densitometric analysis of Ang-2 expression levels. ${ }^{*} P<0.01$; $\#>0.05$. (B) VEGF concentration in the culture media as measured by ELISA. ${ }^{*} P<0.01$.
$120 \%$ increase in heart size suffer from extensive contractile failure (25). Collectively these data suggest that the overall extent of cardiac growth is a critical determinant of the difference between physiological and pathological cardiac hypertrophy and that extensive growth of the heart contributes to contractile dysfunction in this mouse model.

The pathological cardiac hypertrophy that developed from prolonged Akt expression was accompanied by impaired coronary angiogenesis (Figure 5), which is consistent with results of studies correlating pathological cardiac hypertrophy with a reduction in capillary density (33). These findings led us to speculate that impaired coronary angiogenesis could contribute to the contractile dysfunction that occurs following prolonged Akt-mediated heart growth. To test this hypothesis, we utilized a decoy VEGF receptor to attenuate angiogenesis during cardiac growth. Inhibition of coronary angiogenesis during the early physiological growth phase resulted in reduced capillary density, contractile dysfunction, and conversion from physiological to pathological hypertrophy (Figure 7). These phenotypes are consistent with those of cardiomyocytespecific VEGF knockout mice, which exhibit decreased capillary density and contractile dysfunction (34). In contrast, the decoy VEGF receptor had no effect on heart function or capillary density in control hearts. Presumably, in the absence of cardiac growth, normal
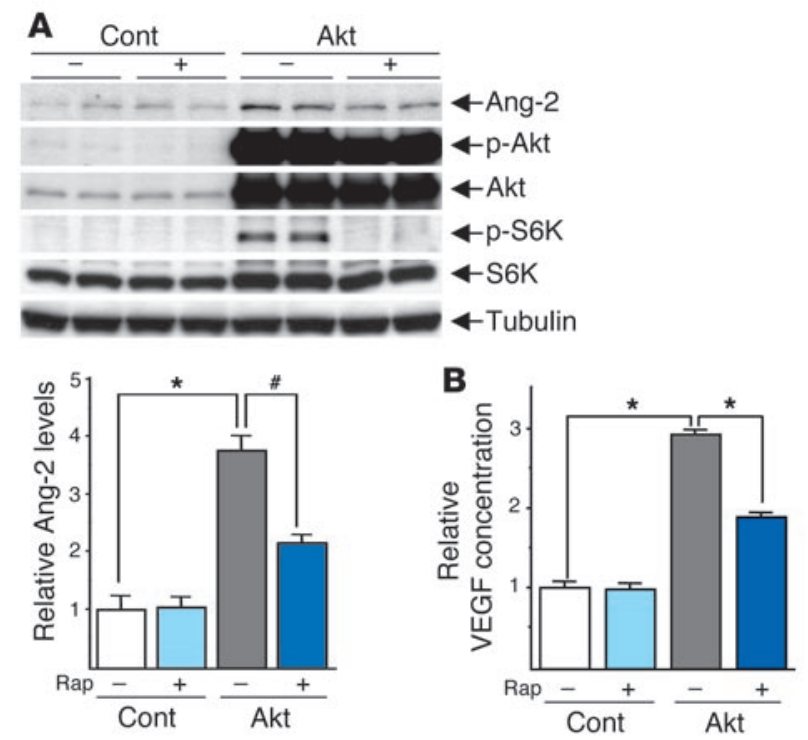
A VEGFR-2/FIk1/KDR
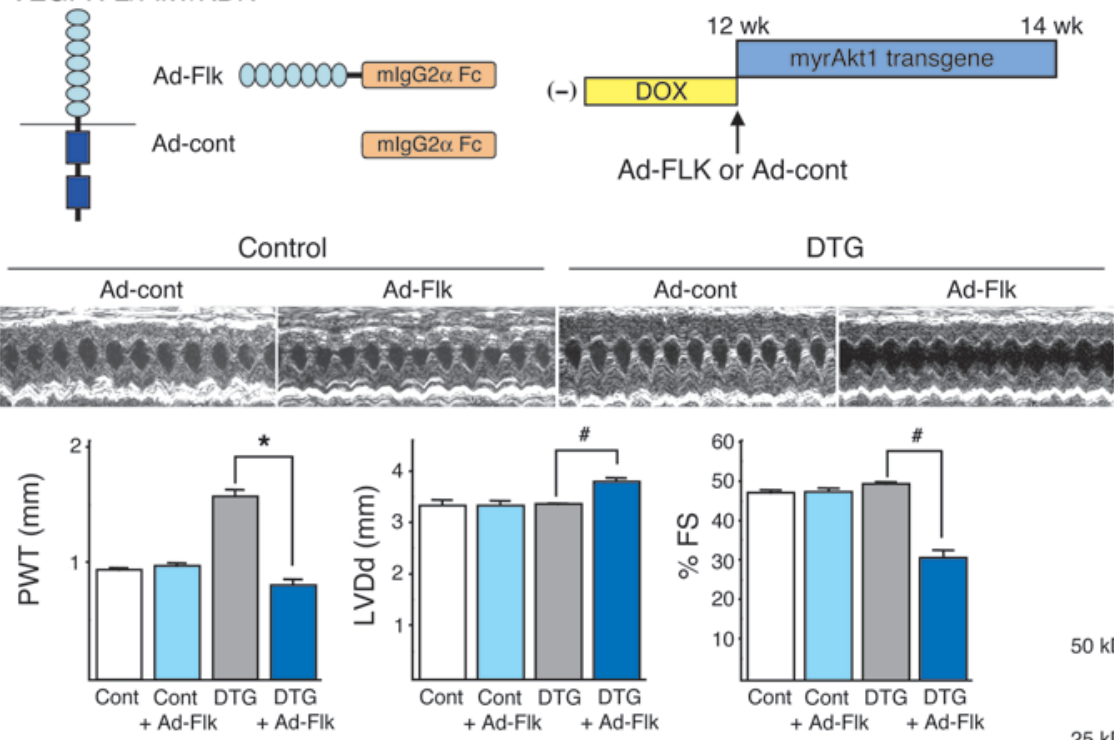

D
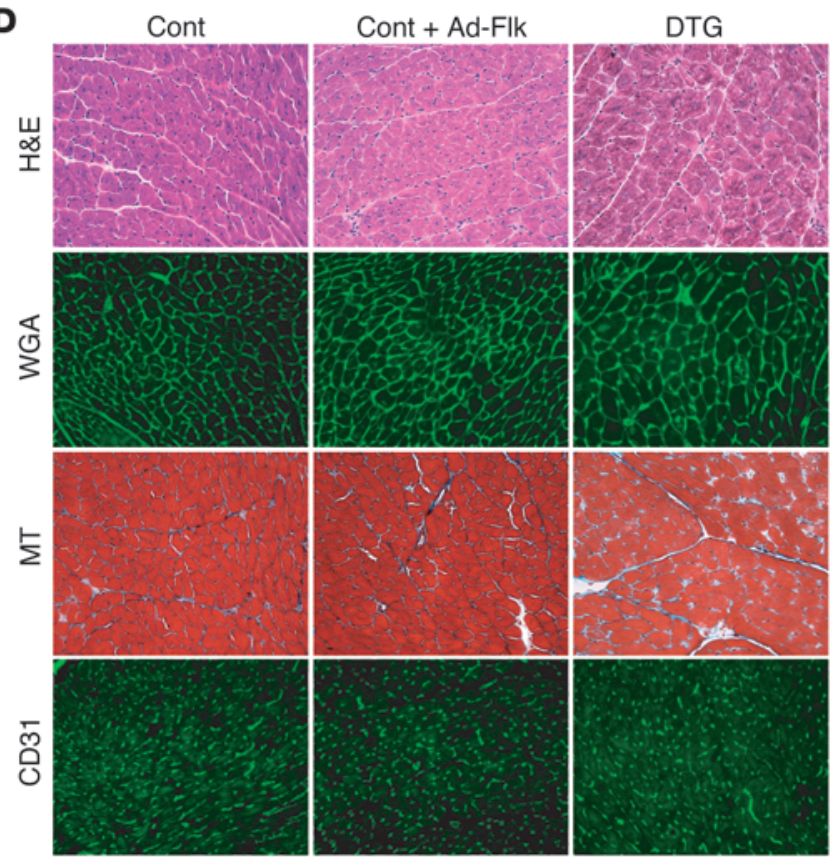

B

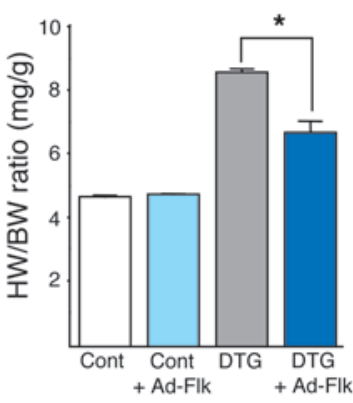

C

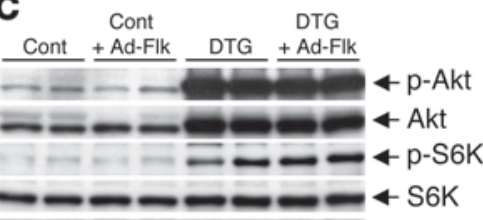

$50 \mathrm{kDa}-$

$25 \mathrm{kDa}-$
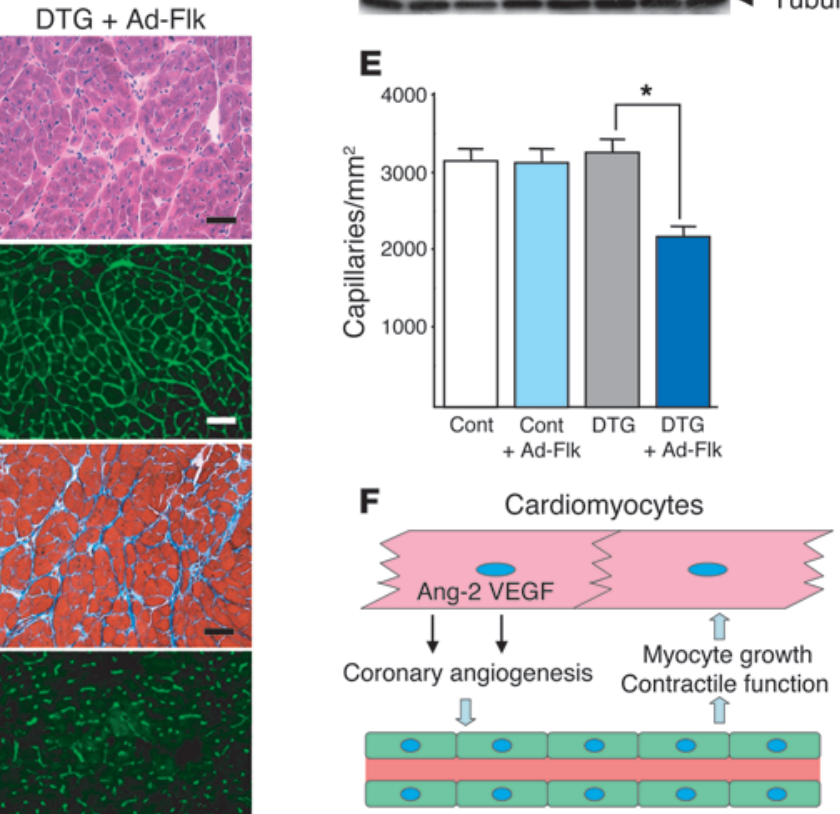

Endothelial cells

Figure 7

Inhibition of coronary angiogenesis results in impaired cardiac growth and contractile dysfunction. (A) Echocardiography. Top: Schematic illustrations of adenoviruses and experimental protocol. KDR, kinase domain insert-containing receptor. Middle: Representative M-mode recordings. Bottom: Echocardiographic parameters. ${ }^{*} P<0.01$; ${ }^{\#} P<0.05$. (B) HW/BW ratio of control or DTG hearts treated with a control vector (Ad-cont) or adenoviral vector encoding Flk1-Fc (Ad-Flk). ${ }^{*} P<0.01$. (C) Representative Western blot of Akt, S6K, VEGF-A, and Ang-2. (D) Histology of control or DTG hearts treated with Ad-cont or Ad-Flk. Scale bars: $50 \mu \mathrm{m}$. (E) Capillary density of control or DTG hearts treated with Ad-cont or Ad-Flk. ${ }^{*} P<0.01$. (F) Cross-talk between cardiac myocytes and coronary vasculature during cardiac growth. Secretion of multiple angiogenic growth factors including VEGF and Ang-2 from cardiomyocytes is thought to be responsible for enhanced coronary angiogenesis during adaptive cardiac growth. Coronary vasculature, on the other hand, is thought to contribute to cardiac growth and the maintenance of contractile function.

contractile capacity can be maintained without the need for ongoing VEGF-dependent angiogenesis. Although we cannot exclude the possibility that the decoy VEGF receptor exerts its negative effects on contractility independently of angiogenesis inhibition, these data support a model in which cardiac function is determined by the relative balance between the extents of cardiac growth and angiogenesis rather than the extent of cardiac growth alone.

Trained athletes frequently exhibit cardiac hypertrophy but not impaired contractility. This may be explained by the fact that a growth in the vascular network accompanies the increase 
in cardiac mass. For example, exercise training in animals is associated with an increase in capillary density and coronary flow reserve (33). In contrast, the pathological hypertrophy seen in patients is typically associated with risk factors, e.g. diabetes, obesity, hypertension, dyslipidemia, and smoking, that contribute to endothelial dysfunction and impaired angiogenic responses. In our mouse model, heart failure developed when myocyte growth and angiogenesis became uncoupled as a result of excessive cardiac hypertrophy. However, in the patient population, impairment in contractile function may not be caused by the extent of cardiac hypertrophy per se, but by the imbalance of tissue growth and angiogenesis in the heart that can be caused by environmental factors that influence endothelial cell function.

The physiological cardiac growth induced by short-term Akt activation was associated with enhanced coronary angiogenesis and increased expression of VEGF-A and Ang-2. Ang-2 is thought to act as a destabilizing signal involved in initiating angiogenic sprouting in the presence of VEGF (35). VEGF-A and Ang-2 are co-induced in or around the vessels undergoing active remodeling process such as angiogenic sprouting in tumors. Furthermore, transgenic coexpression of VEGF-A and Ang-2 in the myocardium exhibits synergistic effects on induction of coronary angiogenesis (36). Because Akt is activated only in cardiac muscle cells in our model system, these data suggest that growth-promoting signals in muscle cells stimulate coronary angiogenesis in a paracrine manner. Our data also show that Akt-mediated induction of VEGF-A and Ang-2 is blocked by rapamycin, which suggests that angiogenic growth factor production is mTOR dependent. Rapamycin prevents the progression of heart failure in the chronic phase in spite of its negative effects on angiogenic growth factor induction presumably because of its negative effect on cardiac growth under these conditions. Since rapamycin does not block VEGF or Ang-2 expression and does not alter capillary density in control hearts (Figure 5, C and D), it appears that basal angiogenic growth factor expression, which is required for the maintenance of coronary vessel homeostasis, is independent of mTOR. Therefore, in the chronically induced state, rapamycin blocks both Akt-mediated hypertrophy and Akt-mediated induction of angiogenic growth factors, resulting in maintained balance between myofiber size and coronary vessels as well as maintained contractility. These data indicate that during heart growth, mTOR functions at a nodal point to coordinate myocyte hypertrophy with blood vessel growth by controlling the production of myocyte-derived angiogenic factors.

While rapamycin has been shown to exert antihypertrophic effects in animal studies, there is no clinical evidence showing that rapamycin diminishes heart size in patients following organ transplant. Several explanations are possible for this apparent discrepancy. First, rapamycin is considered to have little effect on normal heart size and thus would not be expected to reduce heart size in patients without preexisting cardiac hypertrophy. This notion is supported by our observation (Figure 4A) and by previous reports showing that rapamycin does not change heart size in control animals $(37,38)$. Second, when cardiac hypertrophy occurs in transplant patients, it is predominantly induced by pressure overload (hypertension). Unlike in our model, in which hypertrophy is induced by Akt activation alone, in patients, multiple hypertrophic signals including PI3K/Akt, MAPK, calcineurin/nuclear factor of activated T cells (calcineurin/NFAT), and gp130/JAK/STAT pathway are simultaneously activated by pressure overload (39). Because some of these pathways may induce cardiac hypertrophy in an mTOR-independent fashion, rapamycin would only be partially effective in reducing heart size. This notion is supported by studies in animal models showing that rapamycin is only partially effective in attenuating pressure overload-induced hypertrophy $(37,38)$. Third, in clinical trials, rapamycin is typically used in combination with cyclosporine $\mathrm{A}$ (CsA) or other immunosuppressive drugs. It is interesting to note that CsA is reported to attenuate or regress some forms of cardiac hypertrophy in animal models by inhibiting the calcineurin/ NFAT pathway (40), whereas long-term CsA therapy in transplant patients is reported to cause cardiac hypertrophy (41). In humans, chronic CsA therapy induces renal toxicity, leading to hypertension and secondary cardiac hypertrophy. In addition, rapamycin itself may also affect renal function and blood pressure by potentiating the nephrotoxic effects of CsA (42). Therefore, it is possible that hypertension induced either by CsA, rapamycin, or the combination of these agents may mask the antihypertrophic activities of rapamycin in clinical trials. Thus, the data presented in the present article showing that rapamycin can attenuate some forms of cardiac hypertrophy may not apply to pathological hypertrophy in patients. Indeed, further studies would be required to address whether rapamycin has any role in the treatment of cardiac hypertrophy in patients.

Intriguingly, the inhibition of coronary angiogenesis with decoy VEGF receptor attenuated Akt-mediated cardiac growth (Figure 7), which suggests that postnatal cardiac growth is also angiogenesis dependent. It is well established that tumor growth is angiogenesis dependent (43), and adipose tissue mass is also regulated by the vasculature (44). Newly formed coronary vessels may promote cardiac growth and maintain contractile function by carrying nutrients and oxygen into cardiac tissue and removing catabolites and carbon dioxide. It is also possible that the angiogenesis dependence of cardiac growth and function is mediated by paracrine factors (45). For example, it has been shown that normal development of liver or pancreas requires unknown paracrine factor(s) released from endothelial cells $(46,47)$ and hepatocyte growth factor secreted from liver endothelial cells promotes hepatocyte proliferation (48). Since vessel formation during cardiac growth is also dependent on signals from cardiac muscle cells, our study suggests the existence of reciprocal cross-talk mechanisms between the vasculature and cardiac myocytes that regulate coronary angiogenesis, contractile function, and overall heart size (Figure 7F). It should also be noted that $\mathrm{S} 6 \mathrm{~K}$ phosphorylation is not downregulated by decoy VEGF receptor treatment (Figure 7C). Although S6K is a well-established regulator of cell size downstream of Akt/mTOR, this finding suggests that S6K activity does not always correlate with myocyte cell size. This notion is consistent with a recent report demonstrating that deletion of S6K1 and S6K2 does not attenuate cardiac hypertrophy (49). It is also possible that decoy VEGF receptor interferes with signaling pathways downstream of S6K.

In this study, we have used membrane-targeted Akt1 (myrAkt1) as a constitutively active form of Akt 1 expressed in cardiac myocytes as a model system to analyze the role of angiogenesis in heart growth and function. However, myrAkt 1 might behave differently from endogenous Akt1, and, based solely upon the results from this gain-of-function model, one cannot conclude that activation of endogenous Akt 1 results in either physiological or pathological hypertrophy. In this regard, it has been reported that long-term overexpression of nonactivated Akt 1 exclusively in 
the nucleus of cardiomyocytes in $\mathrm{Tg}$ mice protects the heart from ischemia-reperfusion injury without any sign of hypertrophy or heart failure (50). This indicates that Akt exerts distinct effects depending on its subcellular localization and suggests the possibility that the phenotypes of myrAkt 1 overexpression may not represent its functions in cytoplasm or the nucleus. However, while attachment of myristoylated sequence generally results in membrane localization of the protein, it has been shown that myrAkt also localizes to the nucleus and cytoplasm in addition to membrane fraction (51). Consistent with this finding, nuclear forkhead transcription factor FOXO3a is phosphorylated in the hearts of Akt1-Tg mice (52).

The present study provides potential insights about therapeutic strategies for the treatment of heart failure and other human diseases. For example, our study suggests that therapeutic strategies involving the manipulation of angiogenesis may have unexpected consequences on the heart. Specifically, antiangiogenesis therapy for various diseases including cancer (53) could inhibit coronary angiogenesis and have adverse effects on the hearts of patients with cardiac hypertrophy that are at risk for heart failure (54). Because it was found that the imbalance between myocyte growth and coronary angiogenesis plays a critical role in cardiac function, it may be advantageous to stimulate angiogenesis as part of a general strategy to prevent or reverse heart failure. In this regard, our study suggests that it might be possible to treat heart failure with antihypertrophic and proangiogenic agents and that the combined therapy might be more effective than either alone.

\section{Methods}

Cardiac-specific inducible Akt1-Tg mice. HA-tagged mouse myrAkt1 cDNA was subcloned into the HindIII site of pTet-Splice (Invitrogen Corp.). DNA fragment was excised and used for pronuclear injection. Positive founders were identified by PCR and bred to C57BL/ 6 mice to generate stable Tg lines. Primers used for the genotyping were 5 '-CTGGACTACTTGCACTCCGAGAAG- $3^{\prime}$ and $5^{\prime}$-CTGTGTAGGGTCCTTCTTGAGCAG- $3^{\prime}$. $\alpha$ MHC-tTA Tg mice were obtained from the Jackson Laboratory and were crossed with Tet-myrAkt1 mice to generate DTG mice. $\alpha$ MHC-tTA transgene was detected as described previously (29). DTG mice were treated with DOX $(0.5 \mathrm{mg} / \mathrm{ml})$ in drinking water until the predetermined time point for Akt 1 transgene expression, and DOX treatment was restarted to repress the transgene expression. $\alpha M H C-t T A$ single $\mathrm{Tg}$ littermates were used as controls and treated with DOX in the same manner as DTG animals. Rapamycin treatment was performed as described previously (55). Adenovirus vectors encoding Flk1-Fc and control Fc fragment were previously described (32). Intravenous injection of adenoviruses $\left(2 \times 10^{8} \mathrm{PFU}\right)$ was done through carotid veins. The experimental protocol was approved by Boston University School of Medicine's Institutional Animal Care and Use Committee and complied with NIH's Guide for the Care and Use of Laboratory Animals.
Echocardiography. Transthoracic echocardiography was performed with an ACUSON 256 sector scanner (Siemens) equipped with a 13-MHz broadband transducer. All recordings were performed on conscious animals (56).

Western blotting and Akt kinase assay. Western blot analysis was performed as described previously (19). The antibodies used were: phospho-Akt and phospho-p70 S6K from Cell Signaling Technology; Akt1, VP16, p70 S6K, and VEGF-A from Santa Cruz Biotechnology Inc.; HA (12CA5) from Roche Diagnostics Corp.; Ang-2 from Alpha Diagnostic International Inc.; and tubulin from Calbiochem. Akt kinase activity was measured with Akt Kinase Assay Kit from Cell Signaling Technology.

Histological analysis. Heart sections were prepared as described previously (57) and were stained with H\&E for overall morphology, Masson's trichrome for detection of fibrosis, FITC-conjugated wheat germ agglutinin to evaluate myofiber size, and CD31 for determination of capillary density. Anti-CD31 antibody was from Chemicon International.

Real-time PCR. Total RNA was isolated with TRIZOL (Invitrogen Corp.), and CDNA was synthesized using TaqMan Reverse Transcription reagents (Applied Biosystems). Real-time PCR was performed with ABI PRISM 7900 using SYBR Green PCR Master Mix (Applied Biosystems). Transcript levels of ANP and $\beta$-MHC were determined as the number of transcripts relative to those of GAPDH and additionally normalized to the mean value of control hearts. Primers for ANP and $\beta$-MHC were as described previously (58). Primers for GAPDH were 5'-ACTCCACTCACGGCAAATTCA-3' and 5'-GGCCTCACCCCATTTGATG-3'.

Cell culture. Adult rat ventricular myocytes were prepared as described previously (59). Adenoviral vectors encoding constitutively active Akt 1 and control vectors encoding $\beta$-galactosidase were previously described (57), and cultured myocytes were infected with adenovirus vectors overnight on the day of isolation at $50 \mathrm{MOI}$. Cells and culture media were collected 48 hours after virus infection. Cell lysates were subjected to Western blot analysis as previously described (19). VEGF concentration in the culture media was measured using a Mouse VEGF Quantikine ELISA Kit (R\&D Systems).

Statistical analysis. All values are presented as mean \pm SEM. Two-way ANOVA was performed to compare the different parameters among the different groups. For the echocardiographic data, repeated-measures ANOVA was used. A $P$ value less than 0.05 was considered to be statistically significant.

\section{Acknowledgments}

This work was supported by Public Health Service grants AR40197, AG17241, AG15052, HL66957, and HL77774 from the NIH (to $\mathrm{K}$. Walsh) and a Scientist Development Grant from the American Heart Association (to I. Shiojima).

Received for publication February 8, 2005, and accepted in revised form May 17, 2005.

Address correspondence to: Kenneth Walsh, Molecular Cardiology, Whitaker Cardiovascular Institute, Boston University School of Medicine, Boston, Massachusetts 02118, USA. Phone: (617) 414-2392; Fax: (617) 414-2391; E-mail: kxwalsh@bu.edu.
1. Braunwald, E., Colucci, W.S., and Grossman, W. 1997. Clinical aspects of heart failure: high-output heart failure; pulmonary edema. In Heart disease: a textbook of cardiovascular medicine. E. Braunwald, editor. W. B. Sanders. Philadelphia, Pennsylvania, USA. $445-470$.

2. American Heart Association. 2004. Heart diseases and stroke statistics: 2005 update. American Heart Association. Dallas, Texas, USA. http://www.americanheart.org/presenter.jhtml?identifier $=1928$.

3. Olivetti, G., Capasso, J.M., Meggs, L.G., Sonnenblick, E.H., and Anversa, P. 1991. Cellular basis of chronic ventricular remodeling after myocardial infarction in rats. Circ. Res. 68:856-869.

4. Gerdes, A.M., et al. 1992. Structural remodeling of cardiac myocytes in patients with ischemic cardiomyopathy. Circulation. 86:426-430.

5. Levy, D., Garrison, R.J., Savage, D.D., Kannel, W.B., and Castelli, W.P. 1990. Prognostic implications of echocardiographically determined left ventricular mass in the Framingham Heart Study. N. Engl. J. Med. 322:1561-1566.

6. MacLellan, W.R., and Schneider, M.D. 2000. Genetic dissection of cardiac growth control pathways.
Annu. Rev. Physiol. 62:289-319.

7. Molkentin, J., and Dorn, I.G., 2nd. 2001. Cytoplasmic signaling pathways that regulate cardiac hypertrophy. Annu. Rev. Physiol. 63:391-426.

8. Frey, N., and Olson, E.N. 2003. Cardiac hypertrophy: the good, the bad, and the ugly. Annu. Rev. Physiol. 65:45-79.

9. Hudlicka, O., and Brown, M.D. 1996. Postnatal growth of the heart and its blood vessels. J. Vasc. Res. 33:266-287.

10. Richey, P.A., and Brown, S.P. 1998. Pathological versus physiological left ventricular hypertrophy: 
a review. J. Sports Sci. 16:129-141.

11. Verdu, J., Buratovich, M.A., Wilder, E.L., and Birnbaum, M.J. 1999. Cell-autonomous regulation of cell and organ growth in Drosophila by Akt/PKB. Nat. Cell Biol. 1:500-506.

12. Bodine, S.C., et al. 2001. Akt/mTOR pathway is a crucial regulator of skeletal muscle hypertrophy and can prevent muscle atrophy in vivo. Nat. Cell Biol. 3:1014-1019.

13. Tuttle, R.L., et al. 2001. Regulation of pancreatic beta-cell growth and survival by the serine/threonine protein kinase Akt1/PKBalpha. Nat. Med. 7:1133-1137.

14. Chen, W.S., et al. 2001. Growth retardation and increased apoptosis in mice with homozygous disruption of the Akt1 gene. Genes Dev. 15:2203-2208.

15. Cho, H., Thorvaldsen, J.L., Chu, Q., Feng, F., and Birnbaum, M.J. 2001. Akt1/pkbalpha is required for normal growth but dispensable for maintenance of glucose homeostasis in mice. J. Biol. Chem. 276:38349-38352.

16. Cho, H., et al. 2001. Insulin resistance and a diabetes mellitus-like syndrome in mice lacking the protein kinase Akt2 (PKB beta). Science. 292:1728-1731

17. Garofalo, R.S., et al. 2003. Severe diabetes, agedependent loss of adipose tissue, and mild growth deficiency in mice lacking Akt2/PKB $\beta$. J. Clin. Invest. 112:197-208. doi:10.1172/JCI200316885.

18. Peng, X.D., et al. 2003. Dwarfism, impaired skin development, skeletal muscle atrophy, delayed bone development, and impeded adipogenesis in mice lacking Akt1 and Akt2. Genes Dev. 17:1352-1365

19. Shiojima, I., et al. 2002. Akt signaling mediates postnatal heart growth in response to insulin and nutritional status. J. Biol. Chem. 277:37670-37677.

20. Wilkins, B.J., et al. 2004. Calcineurin/NFAT coupling participates in pathological, but not physiological, cardiac hypertrophy. Circ. Res. 94:110-118.

21. Konhilas, J.P., et al. 2004. Sex modifies exercise and cardiac adaptation in mice. Am. J. Physiol. Heart Circ. Physiol. 287:H2768-H2776.

22. Konhilas, J.P., et al. 2005. Loaded wheel running and muscle adaptation in the mouse. Am J. Physiol. Heart Circ. Physiol. doi:10.1152/ajpheart.00085.2005.

23. Naga Prasad, S.V., Esposito, G., Mao, L., Koch, W.J., and Rockman, H.A. 2000. Gbetagamma-dependent phosphoinositide 3-kinase activation in hearts with in vivo pressure overload hypertrophy. J. Biol. Chem 275:4693-4698.

24. Haq, S., et al. 2001. Differential activation of signal transduction pathways in human hearts with hypertrophy versus advanced heart failure. Circulation. 103:670-677.

25. Shioi, T., et al. 2002. Akt/protein kinase B promotes organ growth in transgenic mice. Mol. Cell. Biol. 22:2799-2809.

26. Matsui, T., et al. 2002. Phenotypic spectrum caused by transgenic overexpression of activated Akt in the heart. J. Biol. Chem. 277:22896-22901.

27. Condorelli, G., et al. 2002. Akt induces enhanced myocardial contractility and cell size in vivo in transgenic mice. Proc. Natl. Acad. Sci. U. S. A 99:12333-12338.

28. Taniyama, Y., et al. 2005. Akt3 overexpression in the heart results in progression from adaptive to maladaptive hypertrophy. J. Mol. Cell. Cardiol. 38:375-385.

29. Yu, Z., Redfern, C.S., and Fishman, G.I. 1996. Conditional transgene expression in the heart. Circ. Res. 79:691-697.

30. Harris, T.E., and Lawrence, J.C., Jr. 2003. TOR signaling [review]. Sci. STKE. 2003:re15. doi:10.1126/ stke.2122003re15.

31. Brugarolas, J.B., Vazquez, F., Reddy, A., Sellers, W.R., and Kaelin, W.G., Jr. 2003. TSC2 regulates VEGF through mTOR-dependent and -independent pathways. Cancer Cell. 4:147-158.

32. Kuo, C.J., et al. 2001. Comparative evaluation of the antitumor activity of antiangiogenic proteins delivered by gene transfer. Proc. Natl. Acad. Sci. U. S. A. 98:4605-4610.

33. Hudlicka, O., Brown, M., and Egginton, S. 1992. Angiogenesis in skeletal and cardiac muscle. Physiol. Rev. 72:369-417.

34. Giordano, F.J., et al. 2001. A cardiac myocyte vascular endothelial growth factor paracrine pathway is required to maintain cardiac function. Proc. Natl. Acad. Sci. U. S. A. 98:5780-5785.

35. Yancopoulos, G.D., et al. 2000. Vascular-specific growth factors and blood vessel formation. Nature. 407:242-248.

36. Visconti, R.P., Richardson, C.D., and Sato, T.N. 2002. Orchestration of angiogenesis and arteriovenous contribution by angiopoietins and vascular endothelial growth factor (VEGF). Proc. Natl. Acad. Sci. U. S. A. 99:8219-8224

37. Shioi, T., et al. 2003. Rapamycin attenuates loadinduced cardiac hypertrophy in mice. Circulation. 107:1664-1670.

38. McMullen, J.R., et al. 2004. Inhibition of mTOR signaling with rapamycin regresses established cardiac hypertrophy induced by pressure overload. Circulation. 109:3050-3055.

39. Hoshijima, M., and Chien, K.R. 2002. Mixed signals in heart failure: cancer rules [review]. J. Clin. Invest. 109:849-855. doi:10.1172/JCI200215380.

40. Molkentin, J.D. 2000. Calcineurin and beyond cardiac hypertrophic signaling [review]. Circ. Res. 87:731-738.

41. Ventura, H.O., Malik, F.S., Mehra, M.R., Stapleton, D.D., and Smart, F.W. 1997. Mechanisms of hypertension in cardiac transplantation and the role of cyclosporine. Curr. Opin. Cardiol. 12:375-381.

42. Morales, J.M., Andres, A., Rengel, M., and Rodicio, J.L. 2001. Influence of cyclosporin, tacrolimus and rapamycin on renal function and arterial hypertension after renal transplantation [review]. Nephrol. Dial. Transplant. 16(Suppl. 1):121-124.

43. Folkman, J. 2001. Angiogenesis. In Harrison's principles of internal medicine. 15th edition. B. Eraunwald et al., editors. McGraw-Hill. New York, New York,
USA. 517-530.

44. Rupnick, M.A., et al. 2002. Adipose tissue mass can be regulated through the vasculature. Proc. Natl. Acad. Sci. U. S. A. 99:10730-10735.

45. Folkman, J. 1998. Is tissue mass regulated by vascular endothelial cells? Prostate as the first evidence [review]. Endocrinology. 139:441-442.

46. Matsumoto, K., Yoshitomi, H., Rossant, J., and Zaret, K.S. 2001. Liver organogenesis promoted by endothelial cells prior to vascular function. Science. 294:559-563.

47. Lammert, E., Cleaver, O., and Melton, D. 2001. Induction of pancreatic differentiation by signals from blood vessels. Science. 294:564-567.

48. LeCouter, J., et al. 2003. Angiogenesis-independent endothelial protection of liver: role of VEGFR-1. Science. 299:890-893.

49. McMullen, J.R., et al. 2004. Deletion of ribosomal S6 kinases does not attenuate pathological, physiological, or insulin-like growth factor 1 receptorphosphoinositide 3-kinase-induced cardiac hypertrophy. Mol. Cell. Biol. 24:6231-6240.

50. Shiraishi, I., et al. 2004. Nuclear targeting of Akt enhances kinase activity and survival of cardiomyocytes. Circ. Res. 94:884-891.

51. Adini, I., Rabinovitz, I., Sun, J.F., Prendergast, G.C., and Benjamin, L.E. 2003. RhoB controls Akt trafficking and stage-specific survival of endothelial cells during vascular development. Genes Dev. 17:2721-2732.

52. Skurk, C., et al. 2005. The FOXO3a transcription factor regulates cardiac myocyte size downstream of AKT signaling. J. Biol. Chem. doi:10.1074/jbc. M500528200.

53. Ferrara, N., Hillan, K.J., Gerber, H.P., and Novotny, W. 2004. Discovery and development of bevacizumab, an anti-VEGF antibody for treating cancer. Nat. Rev. Drug Discov. 3:391-400.

54. Ratner, M. 2004. Genentech discloses safety concerns over Avastin [news]. Nat. Biotechnol. 22:1198.

55. Shioji, K., et al. 2002. Overexpression of thioredoxin-1 in transgenic mice attenuates adriamycin-induced cardiotoxicity. Circulation. 106:1403-1409.

56. Yang, X.P., et al. 1999. Echocardiographic assessment of cardiac function in conscious and anesthetized mice. Am. J. Physiol. 277:H1967-H1974.

57. Fujio, Y., Nguyen, T., Wencker, D., Kitsis, R.N., and Walsh, K. 2000. Akt promotes survival of cardiomyocytes in vitro and protects against ischemiareperfusion injury in mouse heart. Circulation. 101:660-667.

58. Hu, P., et al. 2003. Minimally invasive aortic banding in mice: effects of altered cardiomyocyte insulin signaling during pressure overload. Am. J. Physiol. Heart Circ. Physiol. 285:H1261-H1269.

59. Communal, C., Singh, K., Pimentel, D.R., and Colucci, W.S. 1998. Norepinephrine stimulates apoptosis in adult rat ventricular myocytes by activation of the beta-adrenergic pathway. Circulation. 98:1329-1334. 